\title{
Antisense oligonucleotides offer hope to patients with Charcot-Marie-Tooth disease type 1A
}

\author{
Michael E. Shy \\ Department of Neurology, Carver College of Medicine, University of lowa, lowa City, lowa, USA.
}

\begin{abstract}
Charcot-Marie-Tooth disease type 1A (CMT1A) is the most common heritable peripheral neuropathy and results from a duplication on chromosome 17 that results in an extra copy and increased dosage of peripheral myelin protein 22 (PMP22). Zhao et al., in this issue of the JCl, successfully utilized antisense oligonucleotides (ASOs) to reduce PMP22 and ameliorated neuropathy in both mouse and rat models of CMT1A. These data confirm that strategies to reduce PMP22 have potential as effective therapeutic approaches for CMT1A and lay the groundwork for clinical trials in humans afflicted with this chronic, debilitating neurodegenerative disease.
\end{abstract}

\section{Exciting news for CMT1A researchers}

Charcot-Marie-Tooth disease type 1A (CMT1A) is caused by a 1.4-Mb duplication on chromosome 17p11.2, which contains the peripheral myelin protein 22 (PMP22), a protein primarily expressed in myelinating Schwann cells $(1,2)$. In this issue, Zhao et al. treated two rodent models of CMT1A with PMP22-targeting antisense oligonucleotides (ASOs)and showed a $35 \%$ reduction in PMP22 mRNA (3). This reduction was not only adequate in slowing disease progression, but also improved the CMT1A-associated phenotypes in both models. These results are exciting for CMT1A researchers for several reasons. First, CMT1A is the most common inherited neuropathy, affecting 1:5,000 individuals. Second, severity of CMT1A appears to be dependent on PMP22 copy number, as patients with 4 copies of PMP22 have more severe neuropathy than typical CMT1A patients, who have 3 copies of PMP22 (4). Additionally, patients with missense mutations in PMP22 develop neuropathies that are similar to CMT1A (5). Taken together, these data suggest that reducing PMP22 dosage via ASO targeting could be ideal for CMT1A. Interestingly, deletions in the same 1.4-Mb sequence that result in a single copy of PMP22 lead to a distinct disorder termed hereditary neuropathy with liability to pressure palsies (HNPP), which is characterized by potentially debilitating transient episodes of weakness and sensory loss (6). While a $30 \%$ to $50 \%$ reduction of PMP22 mRNA levels achieved by Zhao et al. may be appropriate for CMT1A, further reduction of PMP22 may induce HNPP.

Zhao et al. evaluated the PMP22targeting ASO approach in both the C22 mouse, a severe demyelinating neuropathy model that expresses seven copies of PMP22 from a yeast artificial chromosome, and a CMT1A rat model that expresses only 3 copies of murine Pmp22 and exhibits a milder form of neuropathy (3). Subcutaneous injections of engineered ASO that were selected for potency against human or rat PMP22 were administered weekly. In the mouse model, injections began at 5 weeks of age, when the mice were symptomatic, and continued for 9 weeks. Rats were treated for 12 weeks beginning at 6 weeks of age, just after the animals become symptomatic. As PNS myelination is largely completed in rodents by 30 days of age (7), the ASO treatments were initiated before the appearance of the long-term

Related Article: p. 359

Conflict of interest: M.E. Shy serves as a consultant for Alnylam Pharmaceuticals and Acceleron Pharma.

Reference information: / Clin Invest. 2018;128(1):110-112. https://doi.org/10.1172/JCI98617.

complications of demyelination, such as axonal degeneration. PMP22 mRNA levels reduced in a dose-dependent fashion in both mice and rats by approximately $35 \%$, although reductions as high as $50 \%$ were observed in mice treated with a high dose of $100 \mathrm{mg} / \mathrm{kg}$. The rodents showed improvement as assessed by clinical, physiological, and morphological measurements. In mice, ASO treatment improved grip strength and rotarod performance, which not only did not decline, but increased over baseline. Compound muscle action potential (CMAP) amplitudes are reduced and correlate with disability in patients with CMT1A and are often used as surrogate markers for axonal degeneration (8). Although treatment increased CMAP amplitudes in both mice and rats, the amplitudes did not reach normal levels. Demyelination results in slowing of nerve conduction velocities (NCV), but does not correlate with disease impairment in CMT1A patients and does not always improve with successful treatment of acquired demyelinating neuropathies, such as Guillain-Barré syndrome or chronic inflammatory demyelinating polyradiculoneuropathy (CIDP). Therefore, it was surprising and impressive to see NCV improve to almost normal levels in the ASO-treated animals. Finally, ASO-mediated depletion of PMP22 improved myelination. Taken together, these results clearly demonstrate that ASO therapy improves both severely affected mouse and moderately affected rat models of CMT1A.

The study by Zhou and colleagues also provides additional insight that will be of potential interest to CMT investigators. Biomarkers of disease severity, progression, potential improvement, and target engagement are currently lacking for CMT1A and will be needed to measure efficacy in clinical trials of potential treatments such as ASO. Zhao and colleagues demonstrated that Schwann cells in skin biopsies from CMTA rat footpads contain elevated PMP22 mRNA levels (3). 


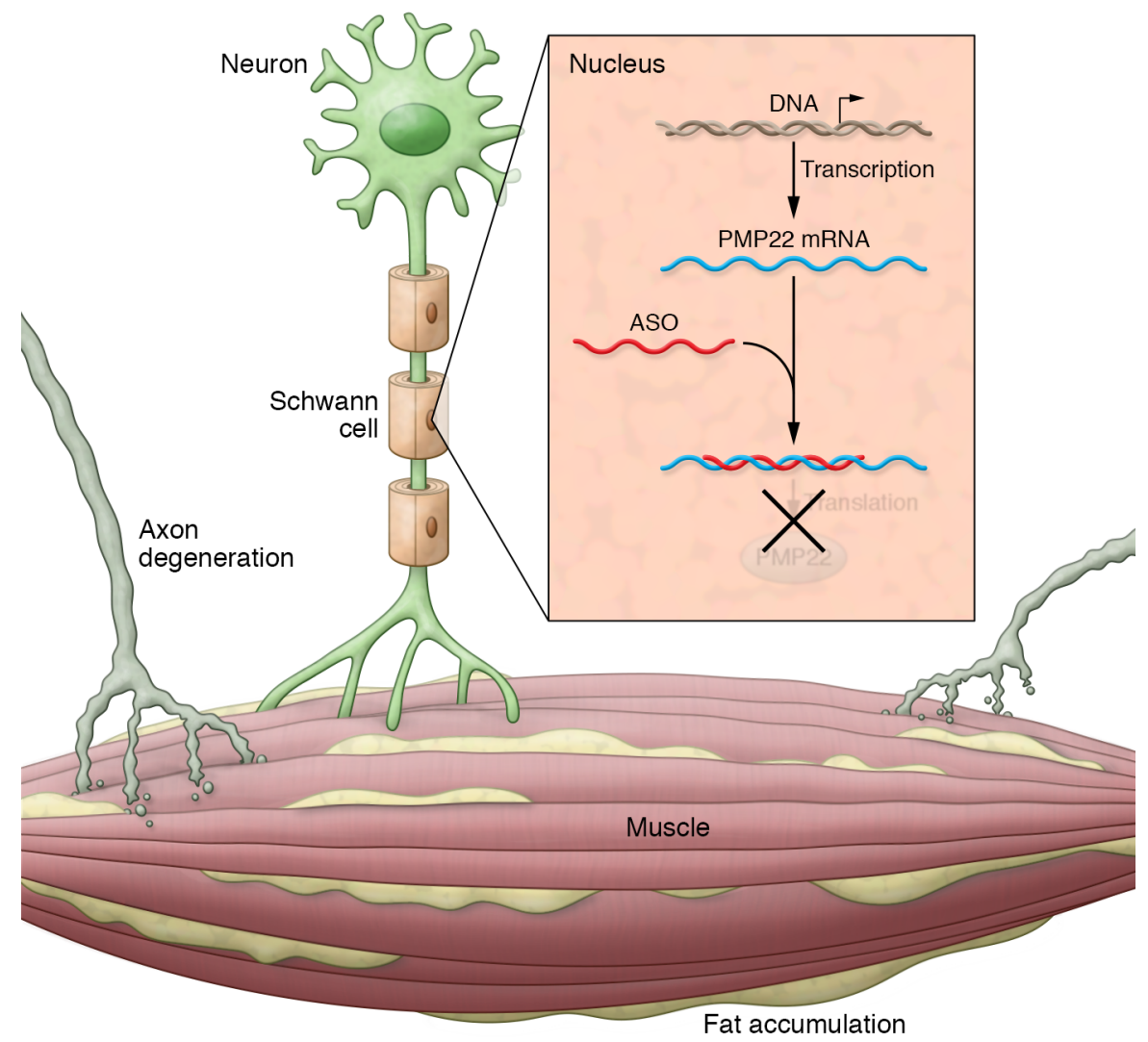

Decreased PMP22 expression in these samples in response to ASO treatment was associated with clinical improvement, suggesting that PMP22 mRNA levels in CMT1A patient skin biopsies could serve as biomarkers of disease and treatment response. Moreover, the RNA-sequencing profiles from C22 mice identified gene-expression patterns that may be important, not only in monitoring treatment effects, but also in understanding disease pathogenesis.

\section{Implications for other neurodegenerative disorders}

ASO therapies have been used for human diseases since 1993 (9) and have demonstrated exciting positive results in humans with debilitating neurodegenerative diseases such as spinal muscular atrophy (SMA) (10) and familial amyloid polyneuropathy (FAP). The SMA splice switching ASO was FDA approved in December 2016 and is currently commercially available to treat patients with various forms of SMA. In a recent trial, the FAP ASO met all its end points, including slowing of neuropathy, although this later benefit was indirect because the ASO targets transthyretin, a serum protein in the liver (ClinicalTrials. gov NCT01737398). An excellent recent review highlights a number of human disorders that are already underway or planned with ASO treatments in patients (11).

The demonstration that ASOs can cross the blood-nerve barrier (BNB) following subcutaneous administration sets the study by Zhao et al. apart. As ASOs are unable to cross the blood-brain barrier (BBB), SMA ASOs are administered intrathecally, and ASO treatments planned for Huntington disease (HD) clinical trials involve either intrathecal or intraventricular administration (reviewed in ref. 11). The $\mathrm{BNB}$ consists of the vascular endoneurium and multilayered perineurium that surrounds nerve fascicles that protect endoneurial components such as myelinating Schwann cells from potentially damaging plasma components and from rapid changes in plasma solute concentrations (12). Prior studies have shown by both immunohistochemistry and mRNA expression that subcutaneous administration of similarly designed ASO can label Schwann cells within the sciatic nerve of mice, though the labeling was weak compared with that of organs not protected by the BNB (13).
Figure 1. ASO therapy for patients with CMT1A causes demyelination that over time leads to secondary axonal degeneration and ff accumulation in the muscle. ASO binding to PMP22 mRNA may alleviate demyelination, but may not reduce axonal degeneration and ff accumulation in muscle that have already occurred, supporting the use of ASO as early as feasible to minimize these secondary complications of CMT1A.

Zhao et al. showed that, at least in rodents, low levels of ASO delivery are adequate for ameliorating CMT1A phenotypes (3).

SMA, FAP, and HD are life-threatening disorders that often lead to death within a few years of symptom onset, while CMT1A is a debilitating disorder that reduces the ability to ambulate without aid or to use hands productively, but does not typically shorten the patient's life (13). Determining the time to initiate and the frequency and duration of ASO treatment is a challenge that will need to be addressed for CMT1A and similar disorders that may require more than 50 years of therapy. Moreover, understanding the best time frame for ASO therapy in CMT1A might eventually provide clues for the treatment of conditions such as SMA, FAP, and HD.

\section{Therapeutic implications}

Several other challenges, including dosage, treatment timing, toxicity, and thrombocytopenia, need to be addressed prior to treating humans with CMT1A. While subcutaneous administration of ASO was effective in rodents, this route may not be adequate in humans. Additionally, ASO labeling was weaker in Schwann cells than in organs outside the BNB in mice (13); therefore, pharmacokinetic (PK) and pharmacodynamic (PD) studies will be essential, as higher dosages in humans may increase toxicity. Alternative administration methods that effectively target Schwann cells and endoneurial compartments in patients may be required to keep systemic dosage low. Recent studies in mouse models of the X-linked form of CMT demonstrated that intrathecal injections of lentiviral vectors can effectively target large numbers of Schwann cells (14). Additionally, it may be critical to determine doses for patients that don't reduce PMP22 levels to less than $50 \%$ of normal, as PMP22 haploinsufficiency may result 
in HNPP. Timing of treatment will also be an important component of treatment. C22 mice and CMT1A rats were treated early, before chronic secondary changes of CMT1A such as axonal degeneration of free fat (ff) replacement in denervated muscle became extensive. ASO specifically reduces $P M P 22$ expression, but does not address these secondary changes that also contribute to disease pathogenesis (Figure 1). Although it will be important to treat older animals that have developed secondary changes, slowing or reversing axonal degeneration and fat formation in muscle might require additional therapeutic strategies for improvement. Safety of treatment is important in chronic diseases that require many years of therapy. Although ASO treatments have proven safe in humans, long-term follow-up is not available in most instances. Thrombocytopenia, a common occurrence after ASO treatment, needs to be monitored during future studies to evaluate ASOs for treatment of CMT1A. While thrombocytopenia following ASO treatments has been mild, transient, and dose dependent and has not led to bleeding abnormalities in animals or humans, on rare occasions, severe thrombocytopenia has been reported during ASO treatment for FAP and familial chylomicronemia syndrome (FCS). Bleeding gums, petechiae, and epistaxis have occurred with ASOs designed to promote exon skipping in Duchenne muscular dystrophy, and death due to intracellular hemorrhage has occurred in patients treated with ASOs for myelofibrosis or recurrent CNS tumors (reviewed in ref. 15). Understanding the basis for thrombocytopenia is an important challenge for the ASO field in general and for CMT1A in particular.

In summary, there remain a number of obstacles to be overcome before ASOs will be routinely used to treat CMT1A. However, the study by Zhao et al. (3) not only offers hope to patients and families with CMT1A, but also offers evidence that another previously untreatable neuromuscular disease is becoming a treatable disorder.

\section{Acknowledgments}

The author receives funding from the National Institute of Neurological Disorders and Stroke/National Center for Advancing Translational Sciences (grant 2U54NS065712), the Muscular Dystrophy Association, and the Charcot-Marie-Tooth Association.

Address correspondence to: Michael E. Shy, 200 Hawkins Drive, Carver College of Medicine, Department of Neurology, University of Iowa, Iowa City, Iowa 52242, USA. Phone: 319.353.5097; Email: michael-shy@uiowa.edu.

1. Lupski JR, et al. DNA duplication associated with Charcot-Marie-Tooth disease type 1A. Cell. 1991;66(2):219-232.

2. Timmerman V, et al. The peripheral myelin protein gene PMP-22 is contained within the Charcot-Marie-Tooth disease type 1A duplication. Nat Genet. 1992;1(3):171-175.

3. Zhao HT, et al. PMP22 antisense oligonucleotides reverse Charcot-Marie-Tooth disease type $1 \mathrm{~A}$ features in rodent models. JClin Invest. 2018;128(1):359-368.

4. Pareyson D, et al. Does CMT1A homozygosity cause more severe disease with root hypertrophy and higher CSF proteins? Neurology. 2003;60(10):1721-1722.
5. Shy M, et al. The hereditary motor and sensory neuropathies: an overview of the clinical, genetic, electrophysiologic and pathlogic features. In: Dyck P, Thomas PK, eds. Peripheral Neuropathy. 4th ed. Philadelphia, Pennsylvania, USA: WB Saunders; 2005:1623-1658.

6. Chance PF, et al. DNA deletion associated with hereditary neuropathy with liability to pressure palsies. Cell. 1993;72(1):143-151.

7. Trapp BD, et al. Cell Biology and myelin assembly. In: Lazzarini RA, ed. Myelin Biology And Disorders. San Diego, Calfornia, USA: Academic Press; 2003:29-56.

8. Krajewski KM, et al. Neurological dysfunction and axonal degeneration in CharcotMarie-Tooth disease type 1A. Brain. 2000; 123(pt 7):1516-1527.

9. Bayever E, et al. Systemic administration of a phosphorothioate oligonucleotide with a sequence complementary to 553 for acute myelogenous leukemia and myelodysplastic syndrome: initial results of a phase I trial. Antisense Res Dev. 1993;3(4):383-390.

10. Finkel RS, et al. Treatment of infantile-onset spinal muscular atrophy with nusinersen: a phase 2, open-label, dose-escalation study. Lancet. 2016;388(10063):3017-3026.

11. Black HA, et al. Corrigendum to "Genetic epidemiology of motor neuron disease-associated variants in the Scottish population." [Neurobiol. Aging 51 (2017) 178.e11-178.e20]. Neurobiol Aging. 2017;56:214.

12. Weerasuriya A. Blood-nerve interface and endoneurial homeostasis. In: Dyck P, Thomas PK, eds. Peripheral Neuropathy. 4th ed. Philadelphia, Pennsylvania, USA: WB Saunders; 2005:651-666.

13. Hung $G$, et al. Characterization of target mRNA reduction through in situ RNA hybridization in multiple organ systems following systemic antisense treatment in animals. Nucleic Acid Ther. 2013;23(6):369-378.

14. Kagiava A, et al. Intrathecal gene therapy rescues a model of demyelinating peripheral neuropathy. Proc Natl Acad Sci U S A. 2016;113(17):E2421-E2429.

15. Chi X, Gatti P, Papoian T. Safety of antisense oligonucleotide and siRNA-based therapeutics. Drug Discov Today. 2017;22(5):823-833. 